\title{
FORMULAÇÃO DE UM NOVO POLÍMERO À BASE PP E PLA
}

\section{ARTIGO ORIGINAL}

FREITAS, Igor Cândido De ${ }^{1}$

SOARES, Bluma Guenther ${ }^{2}$

CORDEIRO, Elisangela Pereira ${ }^{3}$

FREITAS, Igor Cândido De. SOARES, Bluma Guenther. CORDEIRO, Elisangela Pereira. Formulação de um novo polímero à base PP e PLA. Revista Científica Multidisciplinar Núcleo do Conhecimento. Ano 05, Ed. 10, Vol. 17, pp. 137-157. Outubro de 2020. ISSN: 2448-0959, Link de acesso: https://www.nucleodoconhecimento.com.br/engenharia-quimica/novopolimero

\section{RESUMO}

As misturas contendo polipropileno são utilizadas no setor industrial como material de sacola, cadeira, utensílios domésticos, podendo até ser usadas em baterias de celular. O interesse na criação desse material se deve à baixa densidade em relação aos materiais mais comuns como o ferro e o cerâmico, além da boa resistência à corrosão e razoáveis propriedades mecânicas em ambientes sem elevadas temperatura e pressão. Com o objetivo de diversificar as propriedades mecânicas e viscosas e foise usado diferentes tipos de polímeros como o poliácido láctico que apresenta

\footnotetext{
${ }^{1}$ Engenheiro de Materiais pela UFRJ.
}

2 Orientadora. Doutorado em Ciência e Tecnologia de Polímeros. Mestrado em Química. Graduação em Químico.

${ }^{3}$ Coorientadora. Doutorado em andamento em Programa de Pós-graduação em Engenharia Metalúrgica e de Materiais. Mestrado em Ciência e Tecnologia de Polímeros. Especialização em Processamento de Plásticos e Borrachas. Graduação em Licenciatura Plena em Química. 
propriedades benéficas para a reciclagem como a degradação. É um desafio o controle da proporção certa e o uso de aditivos e plastificantes para o controle das propriedades desejadas. Os métodos utilizados para mesurar as propriedades das misturas de polipropileno e poliácido láctico com os diferentes tipos de aditivos, compatibilizantes e plastificantes, foram os ensaios de DMA, TGA, tração, DSC e de reologia seguindo as normas ASTM. Os resultados foram promissores, observandose diferentes propriedades mecânicas e de viscosidade para a mistura PP/PLA/PP-gMA/D nas diversas proporções. Concluindo assim que os diferentes produtos apresentam aplicação variada na indústria.

Palavras-chave: Polipropileno, poliácido láctico, blendas, óleo de linhaça epoxidado.

\section{INTRODUÇÃO}

A preparação de blendas poliméricas, ou seja, misturas poliméricas, torna-se um desafio cada vez maior devido à dificuldade de compatibilidade entre os polímeros. Segundo Utracki (1989) a compatibilização é dependente da estrutura cristalina, ligação química, configuração, fases presentes, entre outros. Para Groeninckx (2006) os materiais de misturas multifases são fortemente dependentes de dois parâmetros: controle da interface e controle da morfologia.

De acordo com Utracki (1989), as misturas poliméricas constituem cerca de 36\% em peso do consumo total de polímero. São muitos os benefícios relacionados como:

(i) Fornecer materiais com propriedades desejadas ao menor preço;

(ii) Entender e aperfeiçoar a performance na engenharia;

(iii) Melhorar propriedades específicas como resistência ao impacto ou resistência aos solventes;

(iv) Dar alternativas para a reciclagem e outros meios de reuso.

Podendo beneficiar o fabricante na; 
(i) Melhoria da processabilidade, um produto uniforme e redução de sucata;

(ii) Mudanças rápidas na formulação;

(iii) Flexibilidade na planta e alta produtividade;

\section{MATERIAIS E MÉTODOS}

Os experimentos foram realizados no laboratório da professora Bluma Guenther Soares, localizado no Instituto de Macromolécula (IMA), Ilha do Governador, Rio de Janeiro

\subsubsection{POLIPROPILENO (PP)}

Cedido pela Braskem $\AA$, código CP442XP (copolímero heterofásico de propeno e eteno com médio índice de fluidez). Especificações na Tabela 1.

Tabela 1: Especificações do PP.

\begin{tabular}{|c|c|c|c|}
\hline & $\begin{array}{l}\text { Método } \\
\text { ASTM }\end{array}$ & Unidade & Valores \\
\hline Índice de Fluidez $\left(230^{\circ} \mathrm{C} / 2,16 \mathrm{~kg}\right)$ & D 1238 & $\mathrm{~g} / 10 \mathrm{~min}$ & 6,0 \\
\hline $\begin{array}{l}\text { Temperatura de Amolecimento Vicat a } 10 \\
\mathrm{~N}\end{array}$ & D 1525 & ${ }^{\circ} \mathrm{C}$ & 145 \\
\hline Alongamento no Escoamento & D 638 & $\%$ & 7 \\
\hline Densidade & D 792 & $\mathrm{~g} / \mathrm{cm}^{3}$ & 0,895 \\
\hline
\end{tabular}

Fonte: Braskem®

\subsubsection{POLIÁCIDO LÁCTICO (PLA)}

Cedido pela Natureworks LLC, código INGEO 2003D (resina termoplástica derivada de recursos renováveis). Especificações na Tabela 2. 
Tabela 2: Propriedades do PLA.

\begin{tabular}{|l|l|l|l|}
\hline & Método de teste & Unidade & Valor \\
\hline Densidade / Gravidade Específica & ASTM D792 & $\mathrm{g} / \mathrm{cm}^{3}$ & 1,24 \\
\hline Índice de Fluidez (230 $\mathbf{~ C / 2 , 1 6 ~} \mathbf{~ k g})$ & ASTM D1238 & $\mathrm{g} / 10 \mathrm{~min}$ & 6,0 \\
\hline $\begin{array}{l}\text { Grade } \\
\text { D-isômero }\end{array}$ & - & - & $\mathrm{L} 96-\mathrm{H}$ \\
\hline Massa Molar Ponderal Média & - & $\%$ & 4 \\
\hline
\end{tabular}

Fonte: Natureworks LLC

\subsubsection{POLIPROPILENO GRAFITIZADO COM ANIDRIDO MALEICO (PP-G-MA)}

Fornecido pela Crompton, código Polybond® 3200. Especificações na Tabela 3.

Tabela 3: Características do PP-g-MA

\begin{tabular}{|l|l|l|}
\hline & Unidade & Valor \\
\hline Índice de Fluidez $\left(\mathbf{1 9 0}{ }^{\circ} \mathbf{C} / \mathbf{2}, \mathbf{1 6} \mathbf{~ k g}\right)$ & $\mathrm{g} / 10 \mathrm{~min}$ & 115 \\
\hline Anidrido Maleico & $\%$ (massa) & 1 \\
\hline Temperatura de Fusão & $\stackrel{\circ}{ } \mathrm{C}$ & 157 \\
\hline
\end{tabular}

Fonte: Crompton

\subsubsection{DRAPEX 8.5}

Concedido pela INBRA INDÚSTRIAS QUÍMICAS LTDA. Especificações na Tabela 4.

Tabela 4: Características do Drapex 8.5.

\begin{tabular}{|l|l|l|l|}
\hline & Método de teste & Unidade & Valor \\
\hline Densidade & - & $\mathrm{g} / \mathrm{cm}^{3}$ & 1,025 \\
\hline Ponto de Fulgor & - & $\stackrel{\circ}{ } \mathrm{C}$ & 460 \\
\hline
\end{tabular}




\begin{tabular}{|l|l|l|l|}
\hline Viscosidade & - & $\mathrm{cP}$ & 300 \\
\hline Índice de Epoxi & $1.10-\mathrm{Q}$ & $\mathrm{g} \mathrm{O} / 100 \mathrm{~g}$ min. & 8,3 \\
\hline Índice de Acidez & $1.3-\mathrm{Q}$ & $\mathrm{mg} \mathrm{KOH} / \mathrm{g}$ máx. & 1,5 \\
\hline
\end{tabular}

Fonte: INBRA INDÚSTRIAS QUÍMICAS LTDA

\subsection{MISTURA REATIVA NA BRABENDER}

\subsubsection{PREPARAÇÃO DA AMOSTRA}

As amostras foram postas na estufa com temperatura de $60 \stackrel{\circ}{ } \mathrm{C}$ para secagem da umidade por aproximadamente 12 horas. Em seguida devidamente pesadas e misturadas de acordo com a proporção de phr baseada na literatura, Tabela 5 (PLOYPETCHARA et al., 2014).

Tabela 5: Proporção das misturas.

\begin{tabular}{|l|l|l|l|l|}
\hline Amostra & PP (phr) & PLA (phr) & PP-g-MA (phr) & D (phr) \\
\hline PP & 100 & - & - & - \\
\hline PLA & - & 100 & - & - \\
\hline PP/PLA & 50 & 50 & - & - \\
\hline PP/PLA/D & 50 & 50 & - & 5 \\
\hline PP/PLA/D & 50 & 50 & - & 10 \\
\hline PP/PLA/PP-g-MA & 50 & 50 & 3 & - \\
\hline PP/PLA/PP-g-MA/D & 50 & 50 & 3 & 5 \\
\hline PP/PLA/PP-g-MA/D & 50 & 50 & 3 & 10 \\
\hline
\end{tabular}

\subsubsection{REALIZAÇÃO DA MISTURA}

Realizado na BRABENDER GmbH \& Co KG ${ }^{\circledR}$. Com temperatura de $190{ }^{\circ} \mathrm{C}$, velocidade de torque das geometrias tipo roller de $60 \mathrm{rpm}$, fator de enchimento 0,75 , volume da cúpula de $55 \mathrm{~cm}^{3}$ e tempo médio de processamento 8 minutos. 


\subsection{PROCESSAMENTO NA MOAGEM}

Realizada a moagem após a mistura reativa na BRABENDER, usando-se o moinho

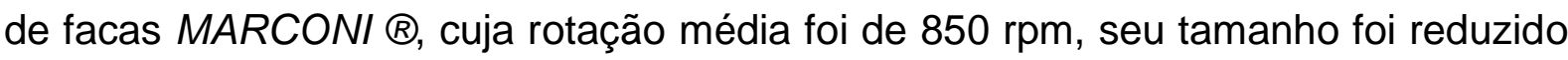
até uma granulometria de $15-25 \mu \mathrm{m}$ segundo dados do fabricante do moinho, na sequência foram ensacadas e etiquetadas.

\subsection{PARÂMETROS DA INJEÇÃO}

\subsubsection{PREPARAÇÃO DAS AMOSTRAS}

As amostras foram postas na estufa à $60{ }^{\circ} \mathrm{C}$ por aproximadamente 12 horas para ocorrer a secagem.

Nessa etapa usou-se a mini injetora Thermo Electron Corporation's HAAKE. MiniJet, para produção de seis corpos de prova, seguindo as normas ASTM D-440 e D-638, sendo a primeira para o teste de DMA e a segunda para o de tração para fazer corpo de prova tipo $\mathrm{V}$. Foram utilizados os seguintes parâmetros de processamento:

- Temperatura de $190^{\circ}$ do cilindro;

- Pressão da injeção de 450 Bar por cinco segundos no molde;

- Recalque de 250 Bar;

- Temperatura do molde em torno de $25 \circ \mathrm{C}$;

- O tempo utilizado para fusão do material no cilindro foi de 5 minutos.

\subsection{PRENSAGEM}

Nessa etapa usou-se material resultante da moagem e serviu para confecção de discos com diâmetro de $25 \mathrm{~mm}$ e $1 \mathrm{~mm}$ de espessura, destinados à reologia. A produção de 4 discos foi feita para cada em moldes de alumínio usando a máquina da Carver Laboratory Press. Parâmetros e sequência de processamento listados abaixo:

1. Temperatura das bases $190^{\circ} \mathrm{C}$, 
2. Pressão de $2,5 \mathrm{kN}$ por um minuto para realizar a degasagem e mitigar os efeitos dos gases.

3. 3 minutos em $5 \mathrm{kN}$.

4. Molde foi retirado e posto na prensa a frio por 4 minutos.

\subsection{TESTE REOLÓGICO}

\subsubsection{PREPARAÇÃO DA AMOSTRA}

O teste de reologia realizado na máquina Discovery Hybrid Rheometer, módulo HR-1, usando os discos feitos na etapa de prensagem, sendo inseridas na placa inferior de uma geometria paralela de $25 \mathrm{~mm}$ de diâmetro e aquecida sob atmosfera de nitrogênio até que ela pudesse fluir.

\subsubsection{PARÂMETROS DO ENSAIO}

Foram usados dois tipos de varreduras, de deformação e de frequência, na primeira a faixa de deformação foi de 0,1-100\% com frequência fixada em $1 \mathrm{~Hz}$ para verificar a faixa do regime viscoelástico enquanto na segunda a frequência variou de 0,1-100 $\mathrm{Hz}$ com deformação fixada em $1 \%$, nos dois casos aplicou-se uma temperatura isotérmica de $190^{\circ} \mathrm{C}$ e o gap entre as placas de $1000 \mu \mathrm{m}$. Os demais parâmetros podem ser observados no anexo 1 seguindo a norma ASTM: D 4440 - 01.

\subsection{EXECUTANDO O ENSAIO DE TRAÇÃO}

\subsubsection{PREPARAÇÃO DA AMOSTRA}

Foram utilizados os corpos de prova produzidos na etapa da injeção, tipo $V$ segundo a norma ASTM D638, a máquina foi ajusta com a distância entre o aperto de $25,4 \mathrm{~mm}$ e a velocidade do deslocamento foi de $5 \mathrm{~mm} \cdot \mathrm{min}^{-1}$, sem uso de extensômetro. 


\subsubsection{REALIZAÇÃO DO ENSAIO E PARÂMETROS}

A máquina utilizada foi da EMIC modelo DL-3000, os parâmetros do teste foram baseados na norma ASTM D638. O Módulo de Young foi calculando escolhendo dois pontos dentro da faixa elástica.

\subsection{ANÁLISE DE CRISTALINIDADE NO DSC}

\subsubsection{PREPARAÇÃO DAS AMOSTRAS}

Os ensaios de DSC foram realizados na máquina DSC 204 F1 Phoenix®, com uma panela de referência já predefinida. No preparo do recipiente foram feitos furos nas tampas de alumina, sentido de dentro para fora, a massa das amostras foram de $10 \pm$ $0,5 \mathrm{mg}$ e de seus respectivos cadinhos ficaram na faixa de $40 \pm 5 \mathrm{mg}$, na sequência colocadas dentro da máquina.

\subsubsection{PARÂMETROS DE ENSAIO}

Etapas de operação enumeradas no apêndice. Utilizou-se como dados o segundo aquecimento e o segundo resfriamento com uma taxa de $10^{\circ} \mathrm{C} / \mathrm{min}$, seguindo a norma ASTM E793 - 06, Tabela 5. O grau de cristalinidade (Xc) foi calculado usando a Equação (1). O fluxo de nitrogênio utilizado para resfriamento está indicado como P2 e PG em ml/min. O grau de cristalinidade (Xc) foi calculado usando a Equação (1):

$$
X_{\mathrm{c}-\mathrm{DSC}}=\frac{\Delta H_{\mathrm{m}}-\Delta H_{\mathrm{cc}}}{\Delta H_{\mathrm{m}}^{0} \times \phi} \times 100 \%
$$

$\Delta \mathrm{H}_{\mathrm{m}}$ e $\Delta \mathrm{H}_{\mathrm{cc}}$ são as entalpias de fusão da mistura e a cristalização a frio do PLA, respectivamente; $\Delta \mathrm{H}_{\mathrm{m}}{ }^{0}$ é a entalpia de fusão teórica do PP $100 \%$ cristalino que equivale a $\left(\Delta \mathrm{H}_{\mathrm{m}} \cdot \mathrm{PP}=138,0 \mathrm{~J} / \mathrm{g}\right)$; e a $\Phi$ é a fração em massa de $(P L A+P P)$ na mistura. 
Tabela 5

\begin{tabular}{|c|c|c|c|c|c|c|c|c|c|c|c|c|c|}
\hline Num & Modo & $\begin{array}{c}\text { Tem } \\
\mathbf{p} \\
\left({ }^{\circ} \mathrm{C}\right)\end{array}$ & $\begin{array}{c}\text { HR } \\
(\mathbf{K} / \mathbf{m i} \\
\mathbf{n})\end{array}$ & $\begin{array}{l}\text { Acq.Ra } \\
\text { te } \\
\text { (pts/mi } \\
\text { n) }\end{array}$ & & $\begin{array}{l}\text { Duraçã } \\
\text { o } \\
\text { (hhrm } \\
\text { m) }\end{array}$ & & & $\begin{array}{l}\text { ST } \\
\text { C }\end{array}$ & & P2:N2 & $\begin{array}{l}\text { PG:N } \\
2\end{array}$ & LN2 \\
\hline- & Início & 20 & & & & 1 & $\begin{array}{l}2 \\
0\end{array}$ & $\begin{array}{l}7 \\
0\end{array}$ & 1 & - & Início & 20 & \\
\hline 1 & $\begin{array}{c}\text { Dinâmic } \\
0\end{array}$ & 200 & 10 & 300 & $\begin{array}{c}00: 1 \\
8\end{array}$ & 1 & $\begin{array}{l}2 \\
0\end{array}$ & $\begin{array}{l}7 \\
0\end{array}$ & 1 & 1 & $\begin{array}{c}\text { Dinâmic } \\
o\end{array}$ & 200 & 10 \\
\hline 2 & Isoterma & 200 & & 50 & $\begin{array}{c}00: 0 \\
5\end{array}$ & 1 & $\begin{array}{l}2 \\
0\end{array}$ & $\begin{array}{l}7 \\
0\end{array}$ & 1 & 2 & Isoterma & 200 & \\
\hline 3 & $\begin{array}{c}\text { Dinâmic } \\
0\end{array}$ & 0 & 10 & 300 & $\begin{array}{c}00: 2 \\
0\end{array}$ & 1 & $\begin{array}{l}2 \\
0\end{array}$ & $\begin{array}{l}7 \\
0\end{array}$ & 1 & 3 & $\begin{array}{c}\text { Dinâmic } \\
0\end{array}$ & 0 & 10 \\
\hline 4 & Isoterma & 0 & & 50 & $\begin{array}{c}00: 0 \\
5\end{array}$ & 1 & $\begin{array}{l}2 \\
0\end{array}$ & $\begin{array}{l}7 \\
0\end{array}$ & 1 & 4 & Isoterma & 0 & \\
\hline 5 & $\begin{array}{c}\text { Dinâmic } \\
\text { o }\end{array}$ & 200 & 10 & 300 & $\begin{array}{c}00: 2 \\
0\end{array}$ & 1 & $\begin{array}{l}2 \\
0\end{array}$ & $\begin{array}{l}7 \\
0\end{array}$ & 1 & 5 & $\begin{array}{c}\text { Dinâmic } \\
\text { o }\end{array}$ & 200 & 10 \\
\hline 6 & Isoterma & 200 & & 50 & $\begin{array}{c}00: 0 \\
2\end{array}$ & 1 & $\begin{array}{l}2 \\
0\end{array}$ & $\begin{array}{l}7 \\
0\end{array}$ & 1 & 6 & Isoterma & 200 & \\
\hline 7 & $\begin{array}{c}\text { Dinâmic } \\
0\end{array}$ & 20 & 10 & 300 & $\begin{array}{c}00: 1 \\
8\end{array}$ & 1 & $\begin{array}{l}2 \\
0\end{array}$ & $\begin{array}{l}7 \\
0\end{array}$ & 1 & 7 & $\begin{array}{c}\text { Dinâmic } \\
0\end{array}$ & 20 & 10 \\
\hline 8 & Isoterma & 20 & & 300 & $\begin{array}{c}00: 0 \\
1\end{array}$ & 1 & $\begin{array}{l}2 \\
0\end{array}$ & $\begin{array}{l}7 \\
0\end{array}$ & $\begin{array}{c}\mathrm{OF} \\
\mathrm{F}\end{array}$ & 8 & Isoterma & 20 & \\
\hline- & Emergência & 210 & & & & & $\begin{array}{l}2 \\
0\end{array}$ & $\begin{array}{l}7 \\
0\end{array}$ & $\begin{array}{c}\mathrm{OF} \\
\mathrm{F}\end{array}$ & - & Emergência & 210 & \\
\hline
\end{tabular}

\subsection{ANÁLISE DE DEGRADAÇÃO NO TGA}

\subsection{PREPARAÇÃO DAS AMOSTRAS}

Nesse teste houve a utilização da máquina TA Instruments Q50, foram utilizadas amostras moídas de massa aproximadamente $10 \pm 2 \mathrm{mg}$.

\subsection{PARÂMETROS}

A faixa de análise foi de 25 até $700{ }^{\circ} \mathrm{C}$ com uma taxa de $20^{\circ} \mathrm{C} / \mathrm{min}$. O tempo de teste foi de 6 horas. Os parâmetros seguiram a norma ASTM E1131. 


\subsection{ANÁLISE TERMO-MECÂNICA NO DMA}

\subsection{PREPARAÇÃO DAS AMOSTRAS}

Os corpos de prova de formato retangulares produzidos na injeção foram cortados até um comprimento de $35 \mathrm{~mm}$.

\subsection{REALIZAÇÃO DO ENSAIO}

A máquina usada foi a $T A$ instruments $D M A$ Q800. A faixa de análise foi de $-50 \stackrel{\circ}{C}$ até $150{ }^{\circ} \mathrm{C}$ com taxa de $3^{\circ} \mathrm{C} / \mathrm{min}$ em atmosfera de nitrogênio de $40 \mathrm{ml} / \mathrm{min}$ e frequência de $1 \mathrm{~Hz}$, seguindo as normas ASTM D4065, D4440 e D5279.

\section{RESULTADOS E DISCUSSÃO}

\subsection{INTERAÇÃO DOS POLÍMEROS}

Figura 1 : Possíveis interações entre os componentes
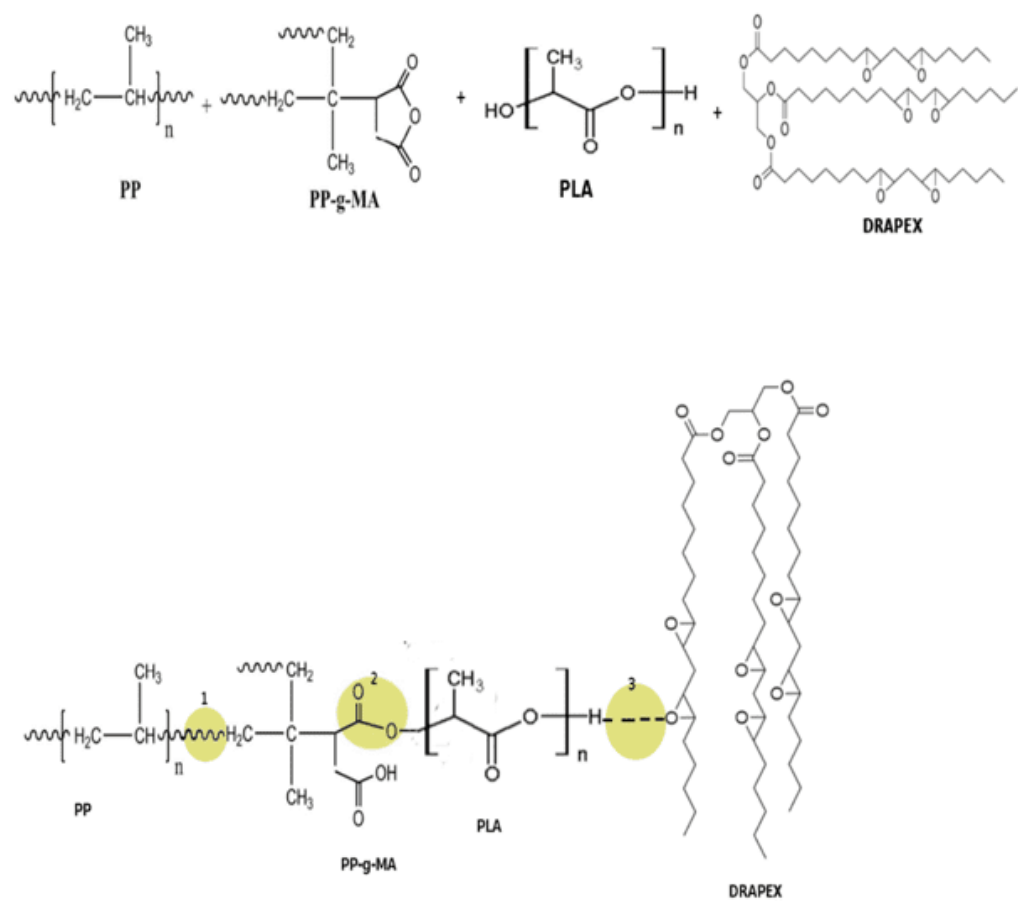

Fonte: (ZANJANIJAM; HAKIMAZIZI, 2016) 
Devido a mistura reativa, ocorre muitas interações entre os polímeros. Uma provável interação entre os polímeros pode ser evidenciada na Figura 1. (ZANJANIJAM; HAKIMAZIZI, 2016).

Em (1) pode-se observar a ligação do PP com PP-g-MA de forma natural e em cadeia; Já em (2) ocorre a quebra do grupo anidrido do PP-g-MA devido à interação com a hidroxila do PLA e ocorrendo assim uma ligação caracterizada por um éter; Enquanto em (3) ocorre ligações de hidrogênio entre o Drapex e o PLA. Outra reação que pode ocorrer em (3) é a quebra do anel epóxi do plastificante e ligando diretamente na cadeia do PLA (AL-MULLA et al., 2010).

\subsection{ENSAIO DE TRAÇÃO}

Tabela 6:Comparativo entre as misturas no ensaio de tração

\begin{tabular}{|c|c|c|c|c|}
\hline Amostra & $\begin{array}{l}\text { Tensão } \\
\text { Max (MPa) }\end{array}$ & $\begin{array}{l}\text { Def. } \\
\text { Max. } \\
(\%)\end{array}$ & $\begin{array}{l}\text { Mod. } \quad \text { De } \\
\text { Elasticidade } \\
\text { (MPa) }\end{array}$ & $\begin{array}{l}\text { Alongamento na } \\
\text { Ruptura (\%) }\end{array}$ \\
\hline PP (100) & $\begin{array}{ll}23,79 \quad \pm \\
0.59 & \end{array}$ & $\begin{array}{l}8,33 \pm \\
0,75\end{array}$ & $951,92 \pm 50,23$ & $1233,33 \pm 20,18$ \\
\hline PLA (100) & $\begin{array}{ll}50,60 \quad \pm \\
3,73 & \end{array}$ & $\begin{array}{ll}5,07 & \pm \\
0,72 & \end{array}$ & $2307,75 \pm 105,76$ & $16,19 \pm 3,14$ \\
\hline PP/PLA (50/50) & $\begin{array}{l}33,31 \quad \pm \\
0,85\end{array}$ & $\begin{array}{l}5,33 \pm \\
0,49\end{array}$ & $1444 \pm 148,2$ & $31,24 \pm 5,23$ \\
\hline $\begin{array}{l}\text { PP/PLA/D } \\
(50 / 50 / 5)\end{array}$ & $\begin{array}{ll}21,00 \quad \pm \\
0,88\end{array}$ & $\begin{array}{ll}5,1 & \pm \\
0,46 & \end{array}$ & $1248 \pm 74,39$ & $42,18 \pm 3,56$ \\
\hline $\begin{array}{l}\text { PP/PLA/D } \\
(50 / 50 / 10)\end{array}$ & $\begin{array}{l}18,44 \quad \pm \\
0,57\end{array}$ & $\begin{array}{l}4,54 \pm \\
0,14\end{array}$ & $1273 \pm 52,41$ & $89,46 \pm 4,12$ \\
\hline $\begin{array}{l}\text { PP/PLA/PP-g-MA } \\
(50 / 50 / 3)\end{array}$ & $\begin{array}{ll}34,02 & \pm \\
1,78 & \end{array}$ & $\begin{array}{l}5,11 \pm \\
0,23\end{array}$ & $1577 \pm 73,32$ & $21,16 \pm 7,05$ \\
\hline $\begin{array}{l}\text { PP/PLA/PP-g- } \\
\text { MA/D (50/50/3/5) }\end{array}$ & $\begin{array}{l}25,01 \quad \pm \\
1,19\end{array}$ & $\begin{array}{l}4,74 \quad \pm \\
0,34\end{array}$ & $1424 \pm 131,29$ & $35,07 \pm 2,14$ \\
\hline
\end{tabular}




\begin{tabular}{|c|c|c|c|c|}
\hline $\begin{array}{l}\text { PP/PLA/PP-g- } \\
\text { MA/D (50/50/3/10) }\end{array}$ & $\begin{array}{l}19,95 \quad \pm \\
046\end{array}$ & $\begin{array}{l}4,92 \pm \\
0,45\end{array}$ & $1200 \pm 97,32$ & $58,14 \pm 4,81$ \\
\hline
\end{tabular}

Fonte: Autor; Origin.

Gráfico 1: Comparativo ensaio de tração

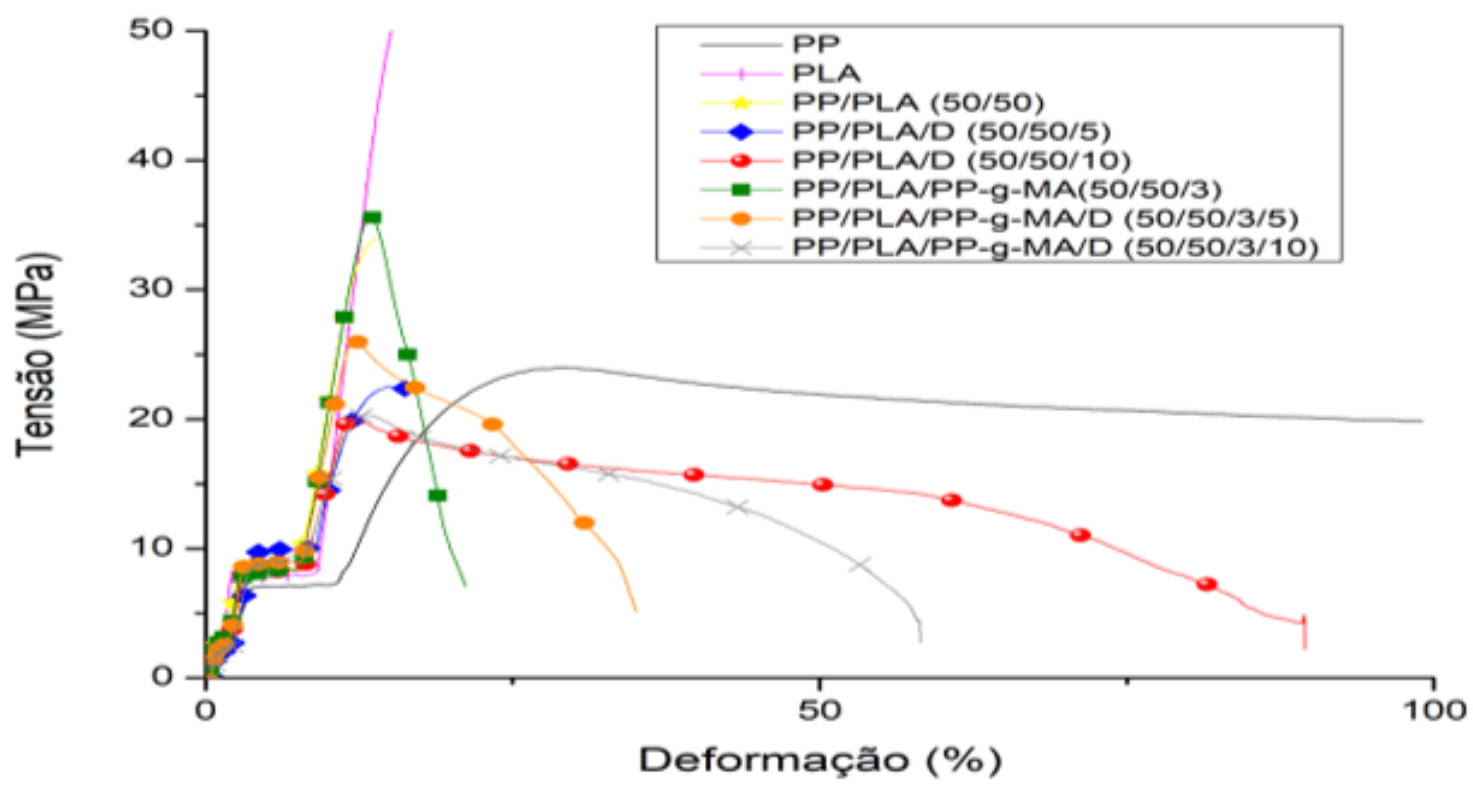

Fonte: Autor; Origin.

No teste de tração, Gráfico 1, as amostras com 10\% de Drapex 8.5 apresentaram maior elongação até ruptura em relação às demais, por exemplo a que continha PPg-MA/D (3/10 phr) chegou até uma elongação de $57 \%$ antes de se romper, enquanto as restantes este valor foi até $30 \%$, a tensão máxima, tabela 7 , foi maior em amostras com PP-g-MA, em torno de $23 \mathrm{MPa}$, o que comprova a compatibilidade e reação do grupo anidrido do PP-g-MA com o PP e PLA, deixando o material resistente. 
Gráfico 2: Comparação do alongamento (esquerda) e Módulo de Elasticidade (direita).
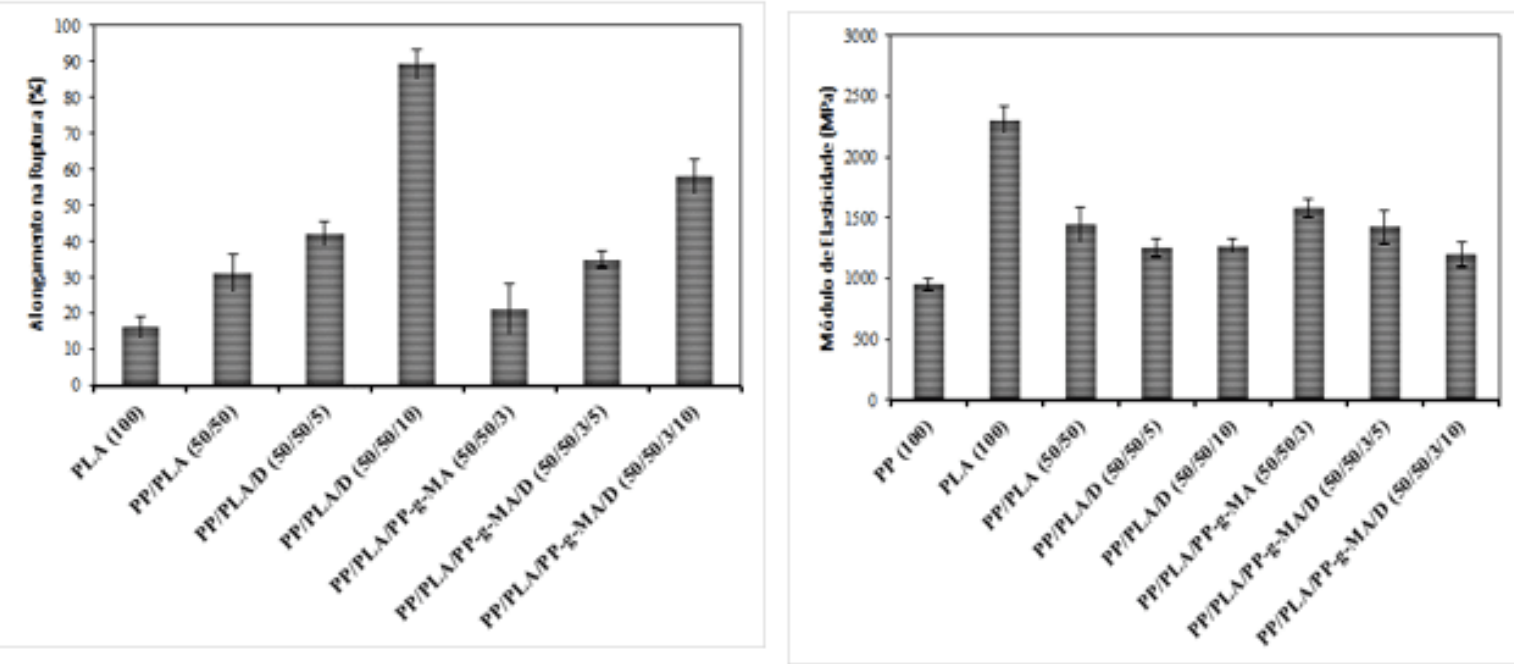

Fonte: Autor; Origin.

Pode-se também observar que houve um decréscimo no módulo de elasticidade para materiais com o plastificante, que pode ter como causa a boa flexibilidade do material. No entanto, os maiores módulos foram para os com PP-g-MA ficando o módulo na faixa de $1500 \mathrm{MPa}$. Um aumento no coeficiente de elasticidade faz o material ser duro e resistente, porém frágil ao impacto (PLOYPETCHARA et al., 2014).

\subsection{REOLOGIA}

Gráfico 3: Comparativo da viscosidade complexa para as misturas

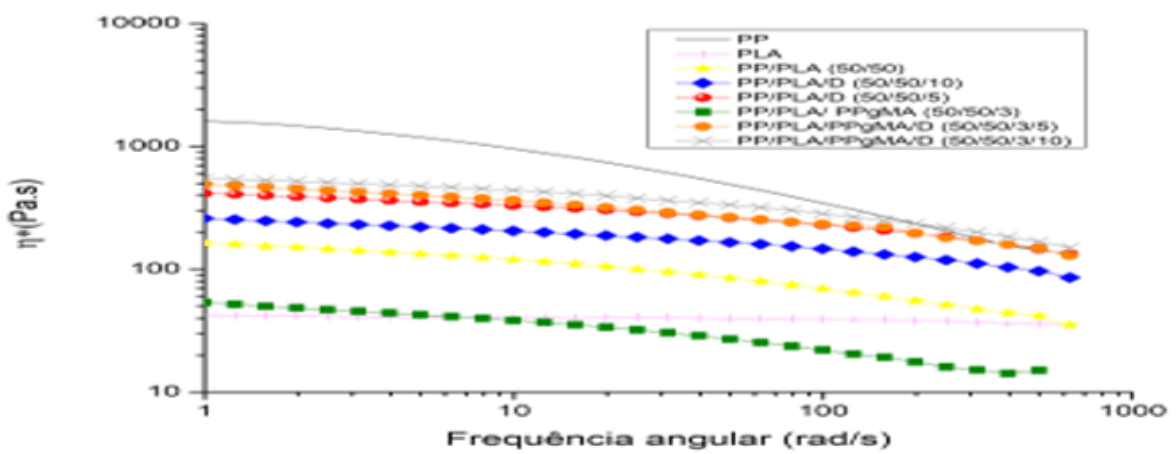

Fonte: Autor; Origin 
Gráfico 4: Comparativo do módulo de armazenamento (esquerda) e módulo de perda (direita)
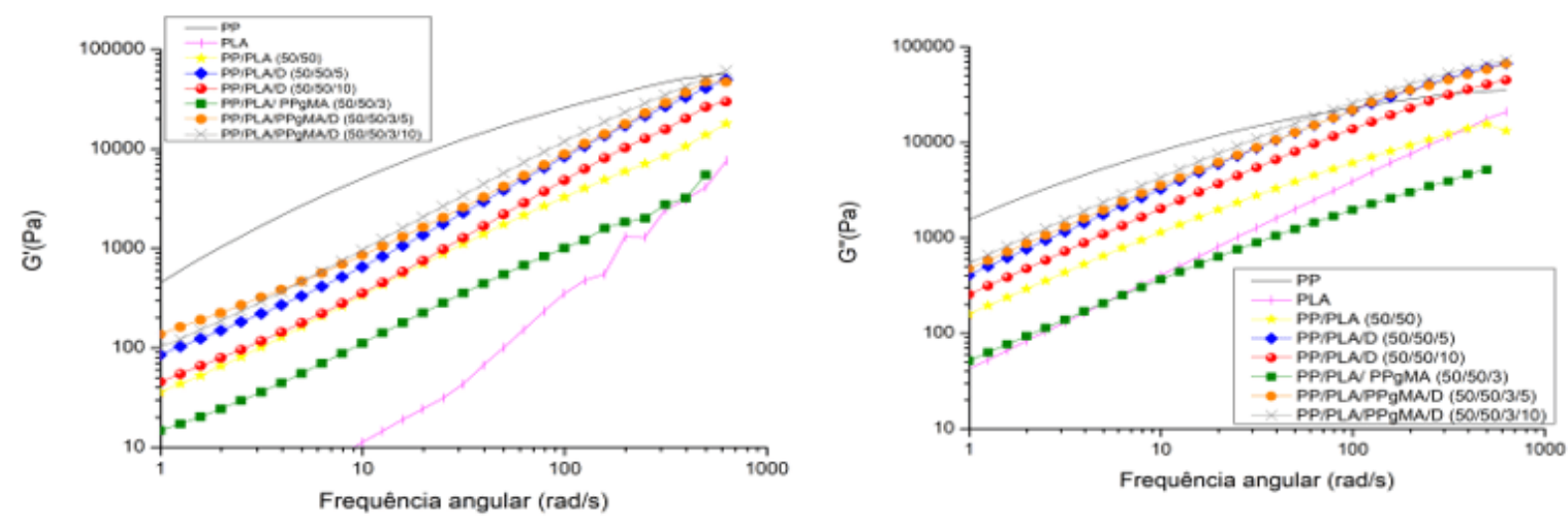

Fonte: Autor; Origin

Pelo Gráfico 3, a diminuição da viscosidade complexa, com o aumento da porcentagem de Drapex 8.5 em misturas de PP/PLA/D, causada pela ação do plastificante aumentando a flexibilidade da cadeia, comprovando a sua eficácia. No entanto, para misturas de PP/PLA/PP-g-MA/D houve um aumento da viscosidade complexa que pode ter sido causada pela interação do grupo lateral do PP-g-MA com o grupamento epóxi do Drapex e junto com o PLA realizando ligação com o PP (PLOYPETCHARA et al., 2014).

Para o modulo de armazenamento, Gráfico 4, as misturas PP/PLA/PP-g-MA/D foram as que apresentaram os maiores valores comprovando a interação entre o Drapex 8.5 e os demais polímeros como consequência da boa compatibilidade. Não refletindo no ensaio de tração devido o teste ser realizado com uma certa frequência e temperatura. O módulo de perda, Gráfico 4, obteve um aumento provocado provavelmente pela flexibilidade da cadeia, facilitando a rotação (PLOYPETCHARA et al., 2014). 


\subsection{DSC}

Gráfico 5: DSC para o segundo aquecimento (esquerda) e resfriamento (direita)
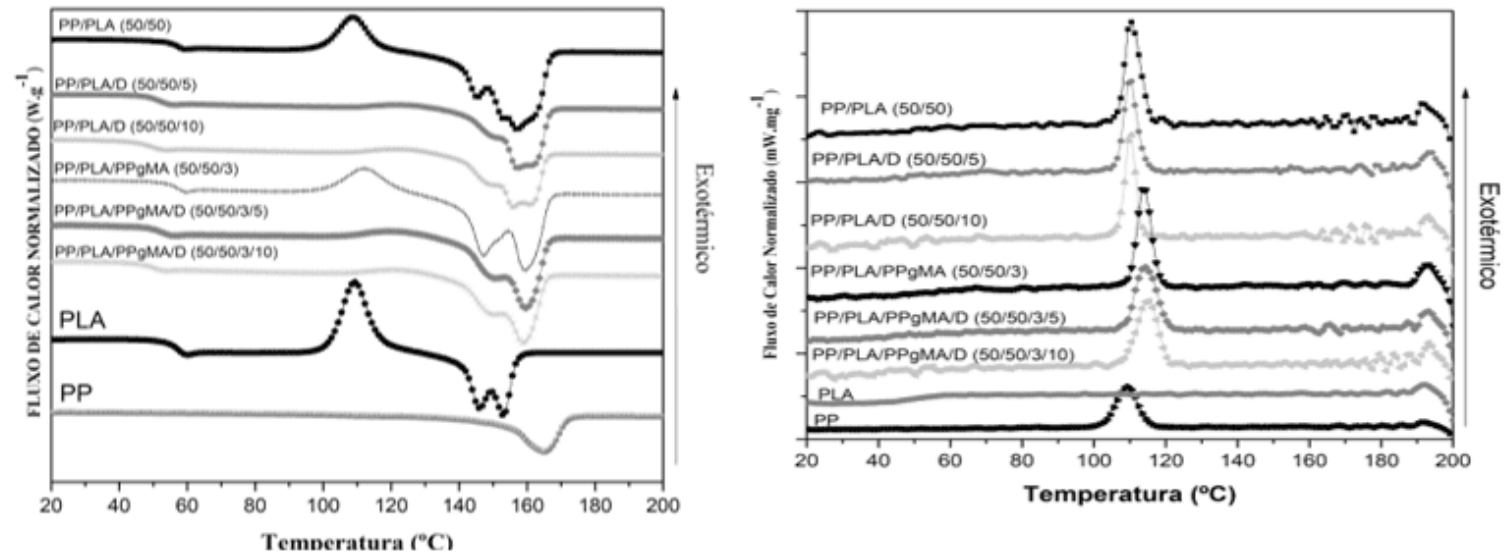

Fonte: Autor; Origin

Tabela 7:Parâmetros de cristalização do fundido da fase PP na blenda ( $2^{\circ}$ resfriamento).

\begin{tabular}{|c|c|c|c|c|c|c|}
\hline Código & $\begin{array}{l}\text { PP } \\
\text { (phr) }\end{array}$ & $\begin{array}{l}\text { PLA } \\
\text { (phr) }\end{array}$ & $\begin{array}{l}\text { PP-g-MA } \\
\text { (phr) }\end{array}$ & $\begin{array}{l}\text { D } \\
\text { (phr) }\end{array}$ & $\begin{array}{l}\Delta \mathrm{H}_{\mathrm{c}} \\
\left(\mathrm{J} \cdot \mathrm{g}^{-1}\right)\end{array}$ & $\begin{array}{l}\text { Tc } \\
\text { (ㅇ) }\end{array}$ \\
\hline PP & 100 & - & - & - & 21,27 & 109,5 \\
\hline PLA & - & 100 & - & - & - & - \\
\hline PP/PLA & 50 & 50 & - & - & 39,31 & 109,1 \\
\hline PP/PLA/D & 50 & 50 & - & 5 & 9,22 & 115,4 \\
\hline PP/PLA/D & 50 & 50 & - & 10 & 29,83 & 110,1 \\
\hline PP/PLA/PP-g-MA & 50 & 50 & 3 & - & 32,40 & 113,7 \\
\hline PP/PLA/PP-g-MA/D & 50 & 50 & 3 & 5 & 32,44 & 114,2 \\
\hline PP/PLA/PP-g-MA/D & 50 & 50 & 3 & 10 & 36,01 & 115,1 \\
\hline
\end{tabular}

Fonte: Máquina DSC 204 F1 Phoenix® 
Tabela 8 :Parâmetros de cristalização a frio da fase PLA na blenda ( $2^{\circ}$ aquecimento).

\begin{tabular}{|c|c|c|c|c|c|c|}
\hline Código & $\begin{array}{l}\text { PP } \\
\text { (phr) }\end{array}$ & $\begin{array}{l}\text { PLA } \\
\text { (phr) }\end{array}$ & $\begin{array}{l}\text { PP-g-MA } \\
\text { (phr) }\end{array}$ & $\begin{array}{l}\text { D } \\
\text { (phr) }\end{array}$ & $\begin{array}{l}\Delta \mathrm{Hcc} \\
\left(\mathrm{J} . \mathrm{g}^{-1}\right)\end{array}$ & $\begin{array}{l}\text { Tcc } \\
\left({ }^{\circ} C\right)\end{array}$ \\
\hline PP & 100 & - & - & - & - & - \\
\hline PLA & - & 100 & - & - & 26,46 & 109,2 \\
\hline PP/PLA & 50 & 50 & - & - & 10,82 & 108,8 \\
\hline PP/PLA/D & 50 & 50 & - & 5 & - & - \\
\hline PP/PLA/D & 50 & 50 & - & 10 & - & - \\
\hline PP/PLA/PP-g-MA & 50 & 50 & 3 & - & 11,56 & 112,0 \\
\hline PP/PLA/PP-g-MA/D & 50 & 50 & 3 & 5 & - & - \\
\hline PP/PLA/PP-g-MA/D & 50 & 50 & 3 & 10 & - & - \\
\hline
\end{tabular}

Fonte: Máquina DSC 204 F1 Phoenix®

Tabela 9:Dados de DSC da mistura PLA-PP e dos componentes puros

\begin{tabular}{|c|c|c|c|c|c|c|c|c|}
\hline Código & $\begin{array}{l}\text { PP } \\
\text { (phr) }\end{array}$ & $\begin{array}{l}\text { PLA } \\
\text { (phr) }\end{array}$ & $\begin{array}{l}\text { PP-g- } \\
\text { MA } \\
\text { (phr) }\end{array}$ & $\begin{array}{l}\text { D } \\
\text { (phr) }\end{array}$ & $\begin{array}{l}\mathrm{Tg} \\
\left({ }^{\circ} \mathrm{C}\right)\end{array}$ & $\operatorname{Tm} \quad\left({ }^{\circ} \mathrm{C}\right)$ & $\begin{array}{l}\Delta \mathrm{Hm} \\
\left(\mathrm{J} \cdot \mathrm{g}^{-1}\right)\end{array}$ & $\begin{array}{l}\text { Xc } \\
(\%)\end{array}$ \\
\hline PP & 100 & - & - & - & - & 166,0 & 21,27 & 15,4 \\
\hline PLA & - & 100 & - & - & 51,1 & 153,0 & 28,03 & 1,7 \\
\hline PP/PLA & 50 & 50 & - & - & 55,3 & 167,1 & 51.0 & 29,1 \\
\hline PP/PLA/D & 50 & 50 & - & 5 & 47,6 & 162,0 & 10,23 & 7,4 \\
\hline PP/PLA/D & 50 & 50 & - & 10 & 46,5 & 155,9 & 34,38 & 24,9 \\
\hline $\begin{array}{l}\text { PP/PLA/PP-g- } \\
\text { MA }\end{array}$ & 50 & 50 & 3 & - & 55,5 & 159,3 & 52,19 & 29,5 \\
\hline $\begin{array}{l}\text { PP/PLA/PP-g- } \\
\text { MA/D }\end{array}$ & 50 & 50 & 3 & 5 & 50,4 & 159,5 & 40,76 & 29,5 \\
\hline $\begin{array}{l}\text { PP/PLA/PP-g- } \\
\text { MA/D }\end{array}$ & 50 & 50 & 3 & 10 & 47,9 & 168,9 & 39,86 & 28,9 \\
\hline
\end{tabular}


Fonte: Máquina DSC 204 F1 Phoenix®

Gráfico 5, as amostras com presença de apenas Drapex levaram a uma ausência do pico cristalino o que está de acordo com a função teórica do plastificante, flexibilizando a cadeia, inviabilizando a cristalização a frio.

Houve o aparecimento de dois picos na faixa de fusão, esse efeito causado pela cristalização a frio do PLA depois da $\mathrm{Tg}$, a presença de dois tipos de cristais (morfológicos alfa e da cristalização a frio beta) pela decomposição da cadeia (FERNANDES et al., 1999).

No teste de DSC para o segundo resfriamento, Gráfico 5, as amostras com Drapex apresentaram uma diminuição da temperatura de cristalização e de transição vítrea junto com os picos da cristalização a frio, o que comprova que o plastificante interagiu com o PP-g-MA e as cadeias de PP.

Com o objetivo de determinar o grau de cristalinidade utilizou-se a segunda varredura de aquecimento.

A entalpia de fusão $\left(\Delta \mathrm{H}_{\mathrm{m}}\right)$ da mistura, Tabela 9 , se refere à área total da temperatura de fusão, incluindo o PLA e o PP. Para o cálculo da cristalinidade, Equação (1), os seguintes critérios foram utilizados:

(i) A mistura é composta de PP/PLA (50/50) (wt\%) e como o PP compõe 50\% em massa da mistura e o grau de cristalinidade do PP puro é maior do que a do PLA,

(ii) $\Delta H$ teórico que foi utilizado no cálculo foi a do $P P\left(\Delta H_{m} \cdot P P=138,0 \mathrm{~J} / \mathrm{g}\right)$;

(iii) A fração volumétrica considerada no cálculo foi 1 (relacionado à mistura 100\%);

(iv) $O X_{c}(\%)$ é o grau de cristalinidade da mistura PLA/PP.

As amostras de PLA exibiram um pico de cristalização a frio e o aparecimento do duplo pico de fusão que pode ser atribuído ao derretimento dos cristais originais e dos formados através da cristalização a frio. (LINGSPRUIELL, 2006). 


\subsection{TGA}

Gráfico 7: Gráfico comparativo de TGA para diferentes misturas
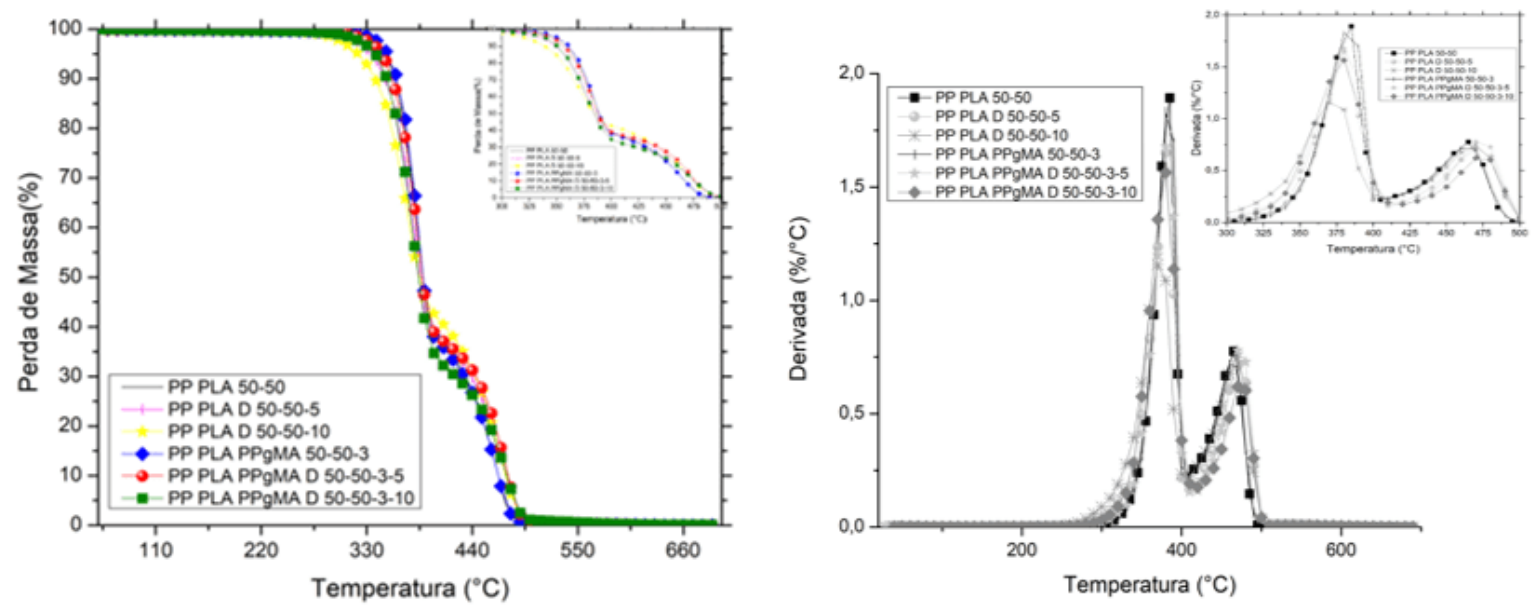

Fonte: Autor; Origin.

Tabela 10: Comparativo dos dados de TGA

\begin{tabular}{|c|c|c|c|c|}
\hline \multirow[b]{2}{*}{ Material } & \multicolumn{4}{|c|}{ Parâmetros } \\
\hline & $\begin{array}{l}\text { On Set } \\
\left({ }^{\circ} \mathrm{C}\right)\end{array}$ & $\begin{array}{l}\text { TMD } \quad(-0 \\
\text { C) }\end{array}$ & $\begin{array}{l}\text { T } 50 \% \quad\left(\frac{0}{}\right. \\
\text { C) }\end{array}$ & $\begin{array}{ll}\text { 1 } & \text { Resíduo } \\
(\%) & \end{array}$ \\
\hline PP PLA (50/50) & 357,21 & 384,89 & 388,98 & 36,35 \\
\hline PP PLA D (50/50/5) & 355,80 & 382,31 & 385,00 & 36,96 \\
\hline PP PLA D (50/50/10) & 341,00 & 373,12 & 384,04 & 41,57 \\
\hline PP PLA PP-g-MA (50/50/3) & 363,20 & 384,70 & 388,60 & 37,49 \\
\hline 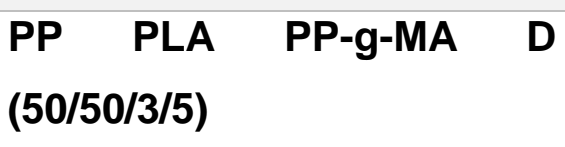 & 360,06 & 384,25 & 387,03 & 37,00 \\
\hline 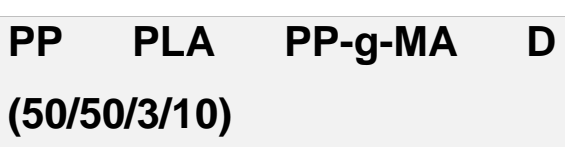 & 352,65 & 379,12 & 383,20 & 41,02 \\
\hline
\end{tabular}

Fonte: TA Analysis e autor

No ensaio de TGA, a amostra PP/PLA/D 50/50/10, Tabela 10 e Gráfico 7, foi a que apresentou um precoce decaimento da curva em relação às demais com temperatura 
On Set de $341^{\circ} \mathrm{C}$, pelo efeito do plastificante. Contudo a mistura PP/PLA/PP-g-MA, foi a que teve o começo de perda de massa postergada para uma temperatura On Set de $\pm 363^{\circ} \mathrm{C}$ (INSTRUMENTALS, 2018).

TMD é a temperatura do pico máximo da derivada, pode-se verificar que está relacionado com a degradação do PLA já que esse é suscetível as mudanças térmicas do que o PP. As misturavas que obtiveram os maiores valores para esse parâmetro foram PP/PLA, PP/PLA/PP-g-MA e PP/PLA/PP-g-MA/D, 384,89, 384,70 e 384,25 respectivamente (INSTRUMENTALS, 2018).

A porcentagem do primeiro resíduo é a primeira derivada da massa pela temperatura, ou seja, o PLA por ter tendência a ser degradável provavelmente se decompôs primeiro e em seguida apresentou uma segunda derivada que é muito provável de ser da massa restante de PP, PP-g-MA e plastificante (INSTRUMENTALS, 2018).

\subsection{DMA}

Gráfico 8: Comparativo de tangente de delta para diferentes misturas

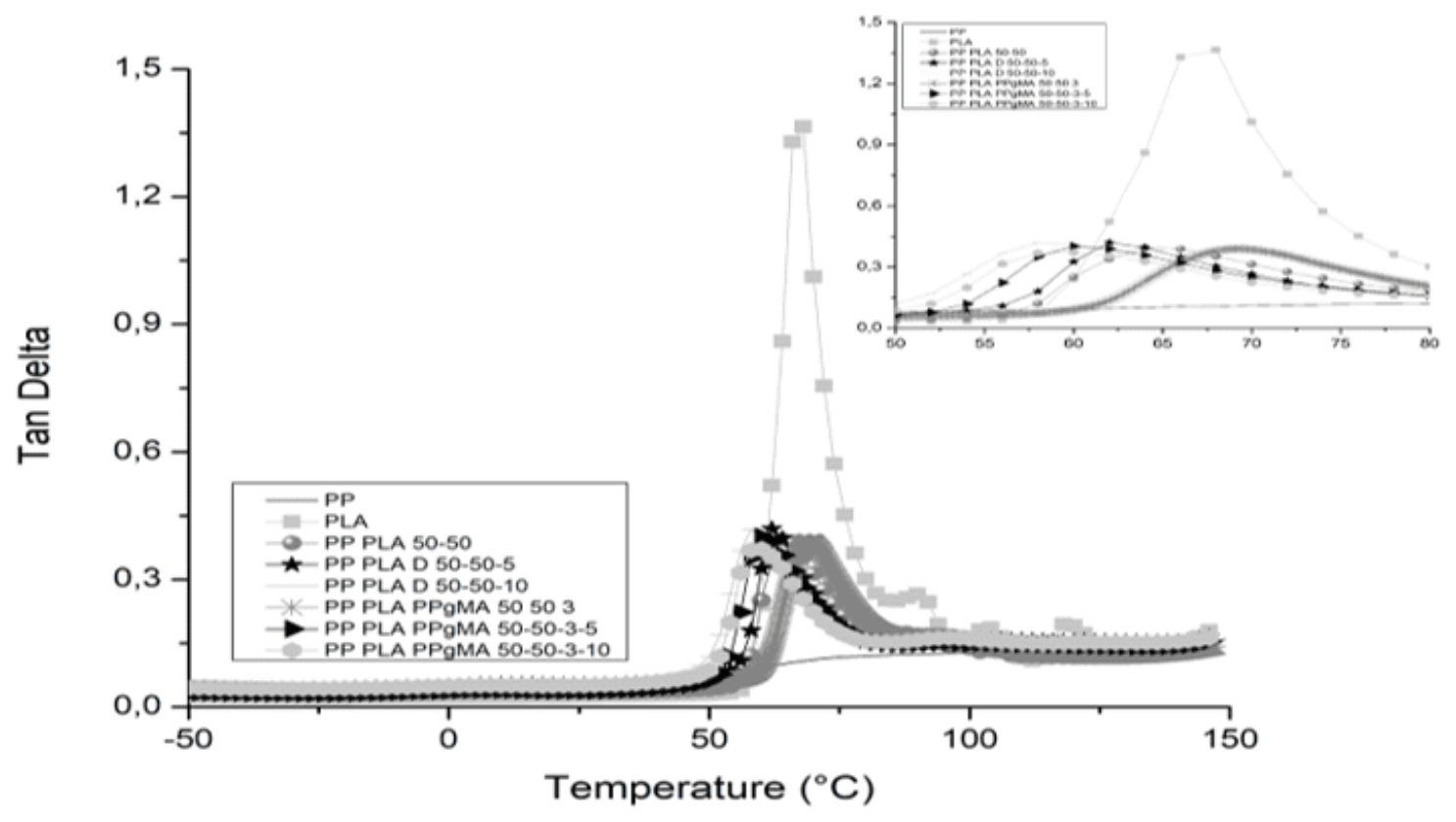

Fonte: Autor; Origin. 
Tabela 11: Comparativo dos dados de DMA

\begin{tabular}{|c|c|c|}
\hline Mistura & E' On set(ํㅜ) & Tan delta $\left({ }^{\circ} \mathrm{C}\right)$ \\
\hline PP & $-8,45$ & $-12,87$ \\
\hline PLA & 57,89 & 66,95 \\
\hline PP PLA 5050 & 56,87 & 64,66 \\
\hline PP PLA D 50505 & 56,33 & 62,34 \\
\hline PP PLA D 505010 & 50,5 & 58,66 \\
\hline PP PLA PP-g-MA 50503 & 63 & 70 \\
\hline PP PLA PP-g-MA D 505035 & 53 & 60,34 \\
\hline PP PLA PP-g-MA D 5050310 & 51,05 & 58,64 \\
\hline
\end{tabular}

Fonte: Autor

No teste de DMA, Gráficos 8 e Tabela 11, o módulo de armazenamento foi maior para a mistura PP/PLA/D (50/50/5) que pode ter sido causado pela interação do grupo epóxi no Drapex com PLA/PP-g-MA tornando o material mais rígido, já o aumento de $5 \mathrm{phr}$ para 10 phr diminui esse efeito. No entanto os materiais só apresentam estável modulo de $\mathrm{E}^{\prime}$ até $50^{\circ} \mathrm{C}$, depois disso o efeito do plastificante faz com que decresça rapidamente. (INSTRUMENTALS, 2018).

Para os valores de Tan $\delta$, pode-se dizer que o material sofreu uma diminuição da temperatura de transição vítrea comprovando o efeito plastificante, aumentando a flexibilidade da cadeia, já as misturas PP/PLA e PP/PLA/PP-g-MA obtiveram os maiores valores de Tan $\delta$, como causa o efeito das ligações entre PP-g-MA Drapex e PLA tornando o material com ligações mais fortes sendo assim menos flexível (INSTRUMENTALS, 2018). 


\subsection{MEV}

Figura 2: a) PP/PLA/D (50/50/5); b) PP/PLA/D (50/50/10); c) PP/PLA/PP-g-MA/D (50/50/3/5); d) PP/PLA/PP-g-MA/D (50/50/3/10); Zoom de 3000X
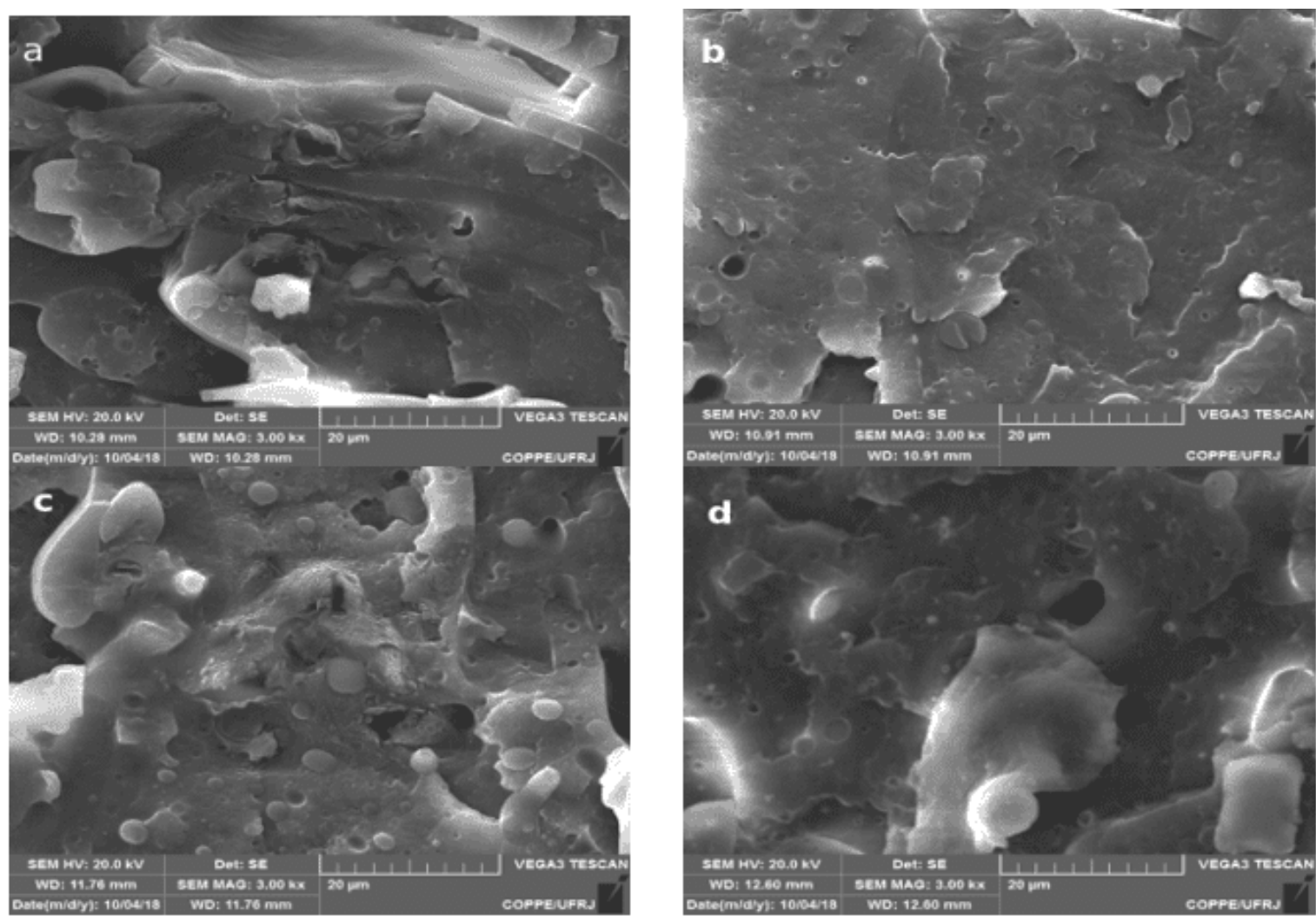

Fonte: Autor; MEV COPPE

Pode-se observar nas amostras a e c, Figura 2, uma grande quantidade de PLA em módulos circulares brancos o que mostra a não compatibilização completa.

Já as amostras b e d os formatos circulares de PLA foram mitigados e a região ficou mais plana evidenciando o aumento da compatibilização com o incremento de Drapex. Portanto houve a compatibilização (CHOUDHARY et al., 2011).

\section{CONCLUSÃO}

A mistura de PP com PLA e PP-g-MA foi promissora, os ensaios reológicos mostraram diversificados resultados para as variadas proporções de mistura. 
Ocorreu aumento do módulo de armazenamento, ou seja, uma maior absorção mecânica com o uso de Drapex 8.5, também aumentou sua flexibilidade que pode ser comprovado na diminuição da transição vítrea vista no ensaio de DMA e no módulo de perda da reologia. Esse material poderia ser utilizado no uso de sacolas plásticas. O que facilita depois a reciclagem por apresentar uma viscosidade menos rígida.

O material ficou mais rígido para misturas sem o plastificante. No entanto, benéfico para fabricação de utensílios como talheres, cadeiras, entre outros.

O polímero resultante obteve boas propriedades térmicas sendo estável até $350 \stackrel{\circ}{\circ}$, o que é um parâmetro tolerado para o clima brasileiro.

O aumento da compatibilidade foi evidenciado no MEV com a diminuição de interfaces circulares de PLA no domínio. Nos ensaios de DSC a mitigação dos picos de cristalização a frio mostrou a perfeita compatibilidade dos polímeros. A taxa de cristalinidade se manteve constante para misturas com PP-g-MA, PP, PLA e D, mostrando mais uma vez o efeito da compatibilização.

Sugestão para trabalho futuro seria implementar carga de CNT no polímero e observar seu comportamento.

\section{REFERÊNCIAS}

AL-MULLA, E. et al., "Properties of epoxidized palm oil plasticized polytlactic acid." Journal of Materials Science. v. 45, n. 7, p. 1942-1946, 2010

AMERICAN SOCIETY FOR TESTING AND MATERIALS. ASTM D4065-12: Standard Practice for Plastics: Dynamic Mechanical Properties: Determination and Report of Procedures. West Conshohocken, PA. ASTM International, 2012.

AMERICAN SOCIETY FOR TESTING AND MATERIALS. ASTM D4440-15: Standard Test Method for Plastics: Dynamic Mechanical Properties Melt Rheology. West Conshohocken, PA. ASTM International, 2015. 
AMERICAN SOCIETY FOR TESTING AND MATERIALS. ASTM D638-14: Standard Test Method for Tensile Properties of Plastics. West Conshohocken, PA. ASTM International, 2014.

AMERICAN SOCIETY FOR TESTING AND MATERIALS. ASTM E1131-08: Standard Test Method for Compositional Analysis by Thermogravimetry. West Conshohocken, PA. ASTM International, 2014.

AMERICAN SOCIETY FOR TESTING AND MATERIALS. ASTM E793-06: Standard Test Method for Enthalpies of Fusion and Crystallization by Differential Scanning Calorimetry. West Conshohocken, PA. ASTM International, 2018

CHOUDHARY, P., et al., "Poly(L-lactide)/polypropylene blends: Evaluation of mechanical, thermal, and morphological characteristics." Journal of Applied Polymer Science, v. 121, n. 6, p. 3223-3237, 2011.

FERNANDES, N., et al., "Thermal decomposition of some chemotherapic substances." Journal of the Brazilian Chemical Society, v. 10, n. 6, p. 459-462, 1999.

INSTRUMENTALS, T., Universal Analysis. 2. ed. New Castle: TA Instruments, 2018.

LING, X., SPRUIELL, J., "Analysis of the complex thermal behavior of poly (L-lactic acid) film. II. Samples crystallized from the melt." Journal of Polymer Science Part B: Polymer Physics, v. 44, n. 23, p. 3378-3391, 2006

PLOYPETCHARA, N., et al., "Blend of Polypropylene/Poly (lactic acid) for Medical Packaging Application: Physicochemical, Thermal, Mechanical, and Barrier Properties." Energy Procedia, v. 56, p. 201-210, 2014.

UTRACKI, L., Polymer alloys and blends, 2 ed., München: Hanser, 1990.

ZANJANIJAM, A., HAKIM, S., AZIZI, H., 2016, "Morphological, dynamic mechanical, rheological and impact strength properties of the PP/PVB blends: the effect of waste PVB as a toughener." RSC Advances, v. 6, n. 50, p. 44673-44686, 2016. 


\section{ANEXO}

\section{Lista de Abreviaturas e Siglas}

\section{Instituição}

IMA- Instituto de Macromolécula;

COPPE- Instituto Alberto Luiz Coimbra de Pós-Graduação e Pesquisa em Engenharia;

\section{Métodos}

DMA - Dynamic Mechanical Analysis; Análise Dinâmico Mecânica.

DSC - Differential scanning calorimetry; Calorimetria Exploratória Diferencial;

DTGA - Differential thermal gravimetric analysis; Derivada primeira da curva TGA.

TGA - Thermogravimetric analysis; Análise termogravimétrica;

\section{Parâmetros}

$\Delta \mathrm{G}$ - Variação da energia livre de Gibbs;

$\Delta \mathrm{H}$ - Variação da entalpia;

$\Delta S$ - Variação da entropia;

IPN - Rede polimérica interpenetrante;

Phr- per hundred of resin- parte por cem de resina.

T - Temperatura absoluta;

Tan- Tangente

Tc - Temperatura de cristalização; 
$\operatorname{Tg}$ - Temperatura de transição vítrea;

Tm - Temperatura de fusão.

\section{Polímeros}

CNT- Carbon nanotubes; Nanotubo de carbono.

D - Drapex $8.5 \AA$ - Óleo epoxidado;

PLA - Poliácido láctico;

PP - Polipropileno;

PP-g-MA - Polipropileno modificado com anidrido maleico;

PS - Poliestireno.

Enviado: Setembro, 2020.

Aprovado: Outubro, 2020. 